\title{
STARS - A Seismic Telescope for Astrophysical Research from Space
}

\author{
Ian W Roxburgh \\ Astronomy Unit, Queen Mary and Westfield College, University of \\ London, Mile End Road, London E1 \&NS, UK.
}

\begin{abstract}
STARS is a proposed asteroseismology (and stellar activity) space mission which is currently under phase A study in the European Space Agency as a candidate for the Medium Size Mission (M3).
\end{abstract}

STARS is a proposed asteroseismology space mission which was submitted to the European Space Agency in May 1993 by a consortium of 31 astronomers (ESA 1993b). It passed the assessment phase (ESA 1994) and is currently under phase A study as one of the short-listed candidates for the European Medium Size Mission (M3). Selection is in 1996. The primary scientific objective is to further our understanding of the evolution of stars by using asteroseismology to probe the interior structure of stars across the Hertzsprung Russell diagram. A further objective is to study stellar activity and surface rotation. STARS is a successor to an earlier proposal, PRISMA, which was one of four short-listed candidates for the M2 mission but it was not selected (ESA 1993a). The primary targets are open clusters with solar type stars brighter than $\mathrm{m}_{v}=10$. The core programme is directed towards a selected group of about 500 stars which is only a very small fraction of the total number of stars (about 20,000 ) that will be observed during the initial 2 year lifetime of the mission. There will be a substantial guest investigator programme.

The proposed instrumentation is a triply reflecting telescope with an effective diameter of $80 \mathrm{~cm}$, a focal ratio of $f / 3$ with a $1^{\circ} \times 1^{\circ}$ field of view, a white light CCD mosaic detector (1024 x 2048), slightly defocused, and operating at a temperature below $-50^{\circ} \mathrm{C}$ (regulated to $0.1^{\circ} \mathrm{C}$ ). This should provide a photometric precision of $0.2 \mathrm{ppm}$ down to $\mathrm{m}_{v}=8$ and $0.8 \mathrm{ppm}$ for $\mathrm{m}_{v}=11$, in $3 \mathrm{~s}$. The total time on each target field will be about one month, or longer, giving an accuracy in frequency determination of solar type stars of the order of 0.1 $-0.3 \mu \mathrm{Hz}$. A photometric precision of $5 \mathrm{ppm}$ can be achieved for stars with $\mathrm{m}_{v}$ $=15$. The maximum number of objects that could be observed in a field of view is about 1,000 giving a total of over 20,000 in the design mission lifetime of 2 years. The mission lifetime could be extended to 5 years. STARS will also carry a Lyman $\alpha$ Monitor, fed by a beam splitter, providing information about stellar rotation and chromospheric activity. The spacecraft will be placed in an Earth-Moon L5 orbit.

The STARS mission will include observations of members of open clusters of different ages, the four target clusters being $\alpha$ Per, the Pleiades, Coma, and the Hyades, but other clusters could be added to this list. The target list will include binaries with good mass and radius determinations, and stars observed 
by HIPPARCOS, for which a distances are well determined. The chosen targets should provide good coverage of the HR diagram including $\delta$ Scuti, roAp, white dwarf, RR Lyrae, $\beta$ Cephei, Am, $\lambda$ Boo and SPB stars. STARS can also be used for microlensing, the detection of stellar companions (possibly planets) and other serendipitous targets. The instrument will be capable of observing simultaneously all stars in a $1^{\circ} \times 1^{\circ}$ field. STARS will remain pointed towards a given field, whether or not it belongs to an open cluster, for at least 1 month, with a duty cycle better than 95 percent.

By devoting 14 months observing the four clusters listed above, and one month on each of 10 different fields, one could observe 120 stars brighter than $\mathrm{m}_{v}=8$ (for which a photometric precision of better than $0.2 \mathrm{ppm}$ will be obtained), and about 150 open cluster members. The observed fields would contain more that 2500 stars brighter than $\mathrm{m}_{v}=11$, for which the expected photometric precision of better than $0.8 \mathrm{ppm}$ will be sufficient to measure solar-like oscillations to a precision of $0.3 \mu \mathrm{Hz}$, and more that 20,000 stars brighter than $\mathrm{m}_{v}=$ 15 , for which a photometric precision of better than $5 \mathrm{ppm}$ will be achievable.

The payload is to be provided by Principal Investigator (PI) teams, funded by ESA Member States. ESA would be responsible for the overall mission and spacecraft design, integration of the payload, launch, operations and transmission of raw data. PIs would have about 30 percent of observing time with 70 percent being available to Guest Investigators (GIs). Data will be available to the general community 1 year after it has been received by the scientist who proposed the observations.

A first Call for Proposals both to PIs and to the community (GIs) would be announced 2 years before launch. A Time Allocation Committee (TAC) would draw up a list of scientific priorities and an Input Consortium would draw up a list of target fields to achieve these goals. The community (GIs) could then propose secondary objectives in each of the fields. It is expected to find between 200 and 1,000 scientifically worthwhile targets per field. Targets need to be selected well in advance (1-2 years) to enable a ground-based supporting programme to be instituted.

\section{References}

ESA 1993a PRISMA, Probing Rotation and Interiors of Stars, Microvariability and Activity. Report on the Phase A Study, European Space Agency ESA SCI(93) 3.

ESA 1993b STARS: Seismic Telescope for Astrophysical Research from Space. Proposal for ESA M3 Mission. ESA 1993.

ESA 1994 STARS: Seismic Telescope for Astrophysical Research from Space. Report of Assessment Study. ESA SCI(94) 8. 